Pacific

Journal of

Mathematics

SOLUTIONS WITH LARGE NUMBER OF PEAKS FOR THE SUPERCRITICAL HÉNON EQUATION

Zhongyuan LiU And ShuangJie Peng 


\title{
SOLUTIONS WITH LARGE NUMBER OF PEAKS FOR THE SUPERCRITICAL HÉNON EQUATION
}

\author{
Zhongyuan LiU And Shuanguie Peng
}

This paper is concerned with the Hénon equation

$$
\begin{cases}-\Delta u=|y|^{\alpha} u^{p+\varepsilon}, u>0, & \text { in } B_{1}(0), \\ u=0 & \text { on } \partial B_{1}(0),\end{cases}
$$

where $B_{1}(0)$ is the unit ball in $\mathbb{R}^{N}(N \geq 4), p=(N+2) /(N-2)$ is the critical Sobolev exponent, $\alpha>0$ and $\varepsilon>0$. We show that if $\varepsilon$ is small enough, this problem has a positive peak solution which presents a new phenomenon: the number of its peaks varies with the parameter $\varepsilon$ at the order $\varepsilon^{-1 /(N-1)}$ when $\varepsilon \rightarrow 0^{+}$. Moreover, all peaks of the solutions approach the boundary of $B_{1}(0)$ as $\varepsilon$ goes to $0^{+}$.

\section{Introduction and main results}

We study the existence of positive solutions to a type of nonlinear elliptic problem whose typical form is the supercritical problem

$$
\begin{cases}-\Delta u=|y|^{\alpha} u^{p+\varepsilon}, u>0, & \text { in } B_{1}(0), \\ u=0 & \text { on } \partial B_{1}(0),\end{cases}
$$

where $p=(N+2) /(N-2), \alpha>0, \varepsilon>0$ and $B_{1}(0)$ is the unit ball in $\mathbb{R}^{N}(N \geq 4)$.

It is well known that the problem

$$
\begin{cases}-\Delta u=|y|^{\alpha} u^{q}, u>0, & \text { in } B_{1}(0), \\ u=0 & \text { on } \partial B_{1}(0),\end{cases}
$$

was proposed by M. Hénon [1973] when he studied rotating stellar structures and is hence called the Hénon equation, and it has attracted a lot of interest in recent years. Ni [1982] first considered (1-2) and proved that it possesses a positive radial solution when $q \in(1,(N+2+2 \alpha) /(N-2))$. Due to the appearance of the weighted term $|y|^{\alpha}$, the classical moving plane method in [Gidas et al. 1979] cannot be applied to problem (1-2). It is natural to ask whether problem (1-2) has nonradial solutions. The existence of a nonradial solution for $1<q<p$ was obtained by

MSC2010: primary 35J60; secondary 35J65, 58E05.

Keywords: peak solutions, supercritical Hénon equation, reduction method. 
Smets, Willem and Su [2002] provided $\alpha$ is large enough. When $q=p-\varepsilon$, Cao and Peng [2003] showed that the ground state solution is nonradial and blows up near the boundary of $B_{1}(0)$ as $\varepsilon \rightarrow 0$. Later on, Peng [2006] constructed multiple boundary peak solutions for problem (1-2). When $q=p$, Serra [2005] proved that problem (1-2) has a nonradial solution provided $\alpha$ is large enough. More recently, Wei and Yan [2013] showed that there are infinitely many nonradial solutions for problem (1-2) with $\alpha>0$. For other results related to Hénon type problems, see [Byeon and Wang 2006; 2005; Cao et al. 2009; Hirano 2009; Pistoia and Serra 2007] and the references therein.

On the other hand, using the Pohozaev identity [1965], we know that for $q \geq$ $\frac{N+2+2 \alpha}{N-2}$ there are no solutions to problem (1-2) in star-shaped domains with respect to the origin. So it seems more interesting to consider whether there are solutions for $q$ in the range $\left(\frac{N+2}{N-2}, \frac{N+2+2 \alpha}{N-2}\right)$. However, much less is known about that case. When $q=\frac{N+2+2 \alpha}{N-2}-\varepsilon$, Gladiali and Grossi [2012] showed that there exists one solution concentrating at $y=0$ provided $0<\alpha \leq 1$. By the results in [Gladiali et al. 2013], the same results still hold when $\alpha$ is not an even integer. In [Li and Peng 2009], the asymptotic behavior of the radial solutions obtained by Ni [1982] was analyzed as $\varepsilon \rightarrow 0^{+}$.

The purpose of this paper is to study the supercritical problem (1-1) and try to construct solutions whose number of peaks varies with $\varepsilon$ as $\varepsilon \rightarrow 0^{+}$. In fact, we will consider the more general problem

$$
\begin{cases}-\Delta u=K(|y|) u^{p+\varepsilon}, u>0, & \text { in } B_{1}(0) \\ u=0 & \text { on } \partial B_{1}(0)\end{cases}
$$

where $K(r) \in C^{1}[0,1]$ and $K(1)>0$.

Without loss of generality, we can assume that

$$
K(1)=1 \text {. }
$$

The main result of this paper is as follows.

Theorem 1.1. Assume that $N \geq 4$. If $K(r)$ satisfies $K(1)>0$ and $K^{\prime}(1)>0$, then there exists $\varepsilon_{0}>0$ such that for $\varepsilon \in\left(0, \varepsilon_{0}\right)$, problem (1-3) has a solution $u_{\varepsilon}$ whose number of local maximal points is of the order $\varepsilon^{-1 /(N-1)}$ as $\varepsilon \rightarrow 0^{+}$. In particular, problem (1-1) has solutions with a large number of peaks for small $\varepsilon>0$.

Remark 1.2. For the case $\alpha=0$, the well-known Pohozaev identity [1965] implies that (1-1) has no solutions for $\varepsilon>0$. It was also shown in [Ben Ayed et al. 2003] that problem (1-1) has no single-peak solutions for $\varepsilon$ small enough. Our results mean that the weight $|y|^{\alpha}$ has a great influence on the existence of peak solutions for problem (1-1). 
Let us outline the main idea in the proof of Theorem 1.1. We introduce some notation first. For $x \in \mathbb{R}^{N}$ and $\Lambda>0$, set

$$
U_{x, \Lambda}(y)=C_{N}\left(\frac{\Lambda}{1+\Lambda^{2}|y-x|^{2}}\right)^{(N-2) / 2}, \quad C_{N}=(N(N-2))^{(N-2) / 4} .
$$

It's well known that $U_{x, \Lambda}(y)$ are the only solutions of

$$
-\Delta u=u^{(N+2) /(N-2)}, u>0, \quad \text { in } \mathbb{R}^{N} .
$$

Let

$$
k=\left[\varepsilon^{-1 /(N-1)}\right],
$$

where $[a]$ denotes the integer part of a real number $a$. By the transformation $u(y) \mapsto \varepsilon^{2 /(4+(N-2) \varepsilon)} u\left(\varepsilon^{1 /(N-2)} y\right)$ and setting $B_{*}=B_{\varepsilon^{-1 /(N-2)}}$, we see that (1-3) becomes

$$
\begin{cases}-\Delta u=K\left(\varepsilon^{1 /(N-2)}|y|\right) u^{p+\varepsilon}, u>0, & \text { in } B_{*}(0), \\ u=0 & \text { on } \partial B_{*}(0) .\end{cases}
$$

We denote by $P U_{x, \Lambda}$, the projection of $U_{x, \Lambda}$, the solution of the problem

$$
\begin{cases}\triangle P U_{x, \Lambda}=\Delta U_{x, \Lambda} & \text { in } B_{*}(0), \\ P U_{x, \Lambda}=0 & \text { on } \partial B_{*}(0) .\end{cases}
$$

Set $y=\left(y^{\prime}, y^{\prime \prime}\right), y^{\prime \prime} \in \mathbb{R}^{N-2}$. Define $\mathcal{H}_{s}=\left\{u: u \in H_{0}^{1}\left(B_{*}(0)\right), u\right.$ is even in $y_{h}, h=2,3, \ldots, N$,

$$
\left.u\left(r \cos \theta, r \sin \theta, y^{\prime \prime}\right)=u\left(r \cos \left(\theta+\frac{2 \pi j}{k}\right), r \sin \left(\theta+\frac{2 \pi j}{k}\right), y^{\prime \prime}\right)\right\} .
$$

Let

$$
x_{j}=\left(r \cos \frac{2(j-1) \pi}{k}, r \sin \frac{2(j-1) \pi}{k}, 0\right), \quad j=1, \ldots, k,
$$

where 0 is the zero vector in $\mathbb{R}^{N-2}$, and let

$$
W_{r, \Lambda}(y)=\sum_{j=1}^{k} P U_{x_{j}, \Lambda} .
$$

In what follows, we always assume that

$$
r \in\left[\varepsilon^{-1 /(N-2)}\left(1-r_{0} \varepsilon^{1 /(N-1)}\right), \varepsilon^{-1 /(N-2)}\left(1-r_{1} \varepsilon^{1 /(N-1)}\right)\right]
$$

for some constants $r_{1}>r_{0}>0$, and that

$$
L_{0} \leq \Lambda \leq L_{1}
$$

for some constants $L_{1}>L_{0}>0$. 
We will prove Theorem 1.1 by verifying the following result.

Theorem 1.3. Under the same assumptions as Theorem 1.1, there exists $\varepsilon_{0}>0$ such that for $\varepsilon \in\left(0, \varepsilon_{0}\right)$, problem (1-4) has a solution $u_{\varepsilon}$ of the form

$$
u_{\varepsilon}=W_{r_{\varepsilon}, \Lambda_{\varepsilon}}+\phi_{\varepsilon}
$$

where $\phi_{\varepsilon} \in \mathcal{H}_{s},\left\|\phi_{\varepsilon}\right\|_{L^{\infty}} \rightarrow 0$ as $\varepsilon \rightarrow 0^{+}, L_{0} \leq \Lambda_{\varepsilon} \leq L_{1}$ and

$$
r_{\varepsilon} \in\left[\varepsilon^{-1 /(N-2)}\left(1-r_{0} \varepsilon^{1 /(N-1)}\right), \varepsilon^{-1 /(N-2)}\left(1-r_{1} \varepsilon^{1 /(N-1)}\right)\right] .
$$

Remark 1.4. In our result, the number of peaks $k$ of the solution $u_{\varepsilon}$ varies with the parameter $\varepsilon$ at the order $\varepsilon^{-1 /(N-1)}$ when $\varepsilon \rightarrow 0^{+}$. This is a new phenomenon for the Hénon equation and is in contrast to the subcritical or critical case. For example, in [Peng 2006], where $\varepsilon<0$, it was proved that for any prescribed integer $k>0$, there exists $\varepsilon_{0}>0$ such that for any $\varepsilon \in\left(-\varepsilon_{0}, 0\right)$, problem (1-4) has a solution which has exactly $k$ peaks.

Remark 1.5. The results of this paper can be considered as a perturbation of those in [Wei and Yan 2013]. In fact the number of bubbles $k$ can be taken to be

$$
k=\left[\delta^{-1 /(N-2)}\right]
$$

for any $|\varepsilon|<\delta \ll 1$. When $\varepsilon=0$, we recover Wei and Yan's result.

We use a reduction argument to prove Theorem 1.3. More precisely, we follow the method in [Wei and Yan 2010b; 2013] to construct peak solutions for problem (1-4). In those papers, where no parameter appears in the considered problem, Wei and Yan used $k$, the number of peaks of the solutions, as the parameter to construct infinitely many positive peak solutions. This idea is very novel and effective for obtaining infinitely many solutions to several types of problems; see [Peng and Wang 2013; Wei and Yan 2010a; 2011]. Unlike the situation in [Wei and Yan 2010b; 2013], here we deal with the supercritical case; we cannot use the variational argument. Instead, we will use the Fredholm theory of compact operators in a suitable Banach space and will employ a direct technique to eliminate the Lagrange multipliers caused from the reduction procedure. Another aspect that differs from [Wei and Yan 2010b; 2013] is that, as we mentioned before, in our proof, we use $\varepsilon$ as the parameter in the construction of peak solutions, but in this paper the number of peaks depends on the parameter $\varepsilon$. As a final remark, we point out that for $\alpha=0$, del Pino, Felmer and Musso [2003] have constructed two-peaked solutions for problem (1-1) in a special domain. Hence, we believe that the effect of the weight $|y|^{\alpha}$ on the existence of solutions is something like that of the domain.

This paper has the following structure. In Section 2, we carry out the finitedimensional reduction procedure. The main results will be proved in Section 3 . We put the energy expansion and some basic estimates used in Sections 2 and 3 in Appendices A and B. 


\section{Finite-dimensional reduction}

In this section, we perform a finite-dimensional reduction. Let

$$
\|u\|_{*}=\sup _{y \in B_{*}(0)}\left(\sum_{i=1}^{k} \frac{1}{\left(1+\left|y-x_{i}\right|\right)^{\frac{1}{2}(N-2)+\tau}}\right)^{-1}|u(y)|
$$

and

$$
\|v\|_{* *}=\sup _{y \in B_{*}(0)}\left(\sum_{i=1}^{k} \frac{1}{\left(1+\left|y-x_{i}\right|\right)^{\frac{1}{2}(N+2)+\tau}}\right)^{-1}|v(y)|,
$$

where $\tau=(N-2) /(N-1)$. We denote by $L_{*}^{\infty}$ and $L_{* *}^{\infty}$ the function spaces defined on $B_{*}(0)$ with finite $\|\cdot\|_{*}$ and $\|\cdot\|_{* *}$ norm, respectively.

Let

$$
Z_{i, 1}=\frac{\partial P U_{x_{i}, \Lambda}}{\partial r}, \quad Z_{i, 2}=\frac{\partial P U_{x_{i}, \Lambda}}{\partial \Lambda} .
$$

First, we consider the linear problem

$$
\left\{\begin{array}{l}
-\Delta \phi-(p+\varepsilon) K\left(\varepsilon^{\frac{1}{N-2}}|y|\right) W_{r, \Lambda}^{p-1+\varepsilon} \phi=h+\sum_{j=1}^{2} c_{j} \sum_{i=1}^{k} U_{x_{i}, \Lambda}^{p-1} Z_{i, j} \text { in } B_{*}(0), \\
\phi \in \mathcal{H}_{s}, \quad\left\langle\sum_{i=1}^{k} U_{x_{i}, \Lambda}^{p-1} Z_{i, l}, \phi\right\rangle=0, \quad l=1,2
\end{array}\right.
$$

for some numbers $c_{i}$, where

$$
\langle u, v\rangle=\int_{B_{*}(0)} u v
$$

Lemma 2.1. Assume there is a sequence $\varepsilon=\varepsilon_{n} \rightarrow 0$ such that $\phi_{\varepsilon}$ solves (2-3) for $h=h_{\varepsilon}$. If $\left\|h_{\varepsilon}\right\|_{* *}$ goes to zero as $\varepsilon$ goes to zero, so does $\left\|\phi_{\varepsilon}\right\|_{*}$.

Proof. The proof of this lemma is very similar to the proof of Lemma 2.1 in [Wei and Yan 2013].

We argue by contradiction. Suppose that there are $\varepsilon \rightarrow 0, h=h_{\varepsilon}, \Lambda_{\varepsilon} \in\left[L_{0}, L_{1}\right]$ and $r_{\varepsilon} \in\left[\varepsilon^{-1 /(N-2)}\left(1-r_{0} \varepsilon^{1 /(N-1)}\right), \varepsilon^{-1 /(N-2)}\left(1-r_{1} \varepsilon^{1 /(N-1)}\right)\right]$ such that $\phi_{\varepsilon}$ solves (2-3) for $h=h_{\varepsilon}, \Lambda=\Lambda_{\varepsilon}, r=r_{\varepsilon}$ with $\left\|h_{\varepsilon}\right\|_{* *} \rightarrow 0$ and $\left\|\phi_{\varepsilon}\right\|_{*} \geq c>0$. Without loss of generality, we may assume that $\left\|\phi_{\varepsilon}\right\|_{*}=1$.

Now rewrite (2-3) in the following integral form:

$$
\begin{aligned}
\phi_{\varepsilon}(y)=(p+\varepsilon) \int_{B_{*}(0)} G_{\varepsilon}(y, z) K\left(\varepsilon^{-1 /(N-2)}|z|\right) W_{r, \Lambda}^{p-1+\varepsilon}(z) \phi_{\varepsilon}(z) d z \\
\quad+\int_{B_{*}(0)} G_{\varepsilon}(y, z)\left(h_{\varepsilon}(z)+\sum_{j=1}^{2} c_{j} \sum_{i=1}^{k} Z_{i, j}(z) U_{x_{i}, \Lambda}^{p-1}(z)\right) d z .
\end{aligned}
$$


By Lemma B.3, we find

$$
\begin{aligned}
\mid(p+\varepsilon) \int_{B_{*}(0)} & G_{\varepsilon}(y, z) K\left(\varepsilon^{-1 /(N-2)}|z|\right) W_{r, \Lambda}^{p-1+\varepsilon}(z) \phi_{\varepsilon}(z) d z \mid \\
& \leq(p+\varepsilon) \int_{B_{*}(0)} \frac{1}{|y-z|^{N-2}} K\left(\varepsilon^{-1 /(N-2)}|z|\right) W_{r, \Lambda}^{p-1+\varepsilon}(z)\left|\phi_{\varepsilon}(z)\right| d z \\
& \leq C\left\|\phi_{\varepsilon}\right\|_{*} \int_{B_{*}(0)} \frac{1}{|y-z|^{N-2}} W_{r, \Lambda}^{p-1+\varepsilon} \sum_{j=1}^{k} \frac{1}{\left(1+\left|z-x_{j}\right|\right)^{\frac{1}{2}(N-2)+\tau}} d z \\
& \leq C\left\|\phi_{\varepsilon}\right\|_{*} \sum_{j=1}^{k} \frac{1}{\left(1+\left|z-x_{j}\right|\right)^{\frac{1}{2}(N-2)+\tau+\vartheta}} .
\end{aligned}
$$

It follows from Lemma B.2 that

$$
\begin{aligned}
\left|\int_{B_{*}(0)} G_{\varepsilon}(y, z) h_{\varepsilon}(z) d z\right| & \leq \int_{B_{*}(0)} \frac{1}{|y-z|^{N-2}}\left|h_{\varepsilon}(z)\right| d z \\
& \leq C\left\|h_{\varepsilon}\right\|_{* *} \sum_{j=1}^{k} \frac{1}{\left(1+\left|z-x_{j}\right|\right)^{\frac{1}{2}(N-2)+\tau}}
\end{aligned}
$$

and

$$
\left|\int_{B_{*}(0)} G_{\varepsilon}(y, z) \sum_{j=1}^{k} Z_{i, l}(z) U_{x_{i}, \Lambda}^{p-1}(z) d z\right| \leq C \sum_{j=1}^{k} \frac{1}{\left(1+\left|z-x_{j}\right|\right)^{\frac{1}{2}(N-2)+\tau}} .
$$

Next, we estimate $c_{\ell}, \ell=1,2$. Multiplying (2-3) by $Z_{1, t}$ and integrating, we obtain that $c_{\ell}$ satisfies

$$
\begin{aligned}
& \sum_{\ell=1}^{2} \sum_{i=1}^{k}\left\langle U_{x_{i}, \Lambda}^{p-1} Z_{i, \ell}, Z_{1, t}\right\rangle c_{\ell} \\
& \quad=\left\langle-\Delta \phi_{\varepsilon}-(p+\varepsilon) K\left(\varepsilon^{-1 /(N-2)}|y|\right) W_{r, \Lambda}^{p-1+\varepsilon} \phi_{\varepsilon}, Z_{1, t}\right\rangle-\left\langle h_{\varepsilon}, Z_{1, t}\right\rangle .
\end{aligned}
$$

It follows from Lemma B.1 that

$$
\begin{aligned}
\left|\left\langle h_{\varepsilon}, Z_{1, \ell}\right\rangle\right| & \leq C\left\|h_{\varepsilon}\right\|_{* *} \int_{\mathbb{R}^{N}} \frac{1}{\left(1+\left|z-x_{1}\right|\right)^{N-2}} \sum_{j=1}^{k} \frac{1}{\left(1+\left|z-x_{j}\right|\right)^{\frac{1}{2}(N+2)+\tau}} d z \\
& \leq C\left\|h_{\varepsilon}\right\|_{* *} .
\end{aligned}
$$

On the other hand, using Lemma B.3, we obtain

$$
\begin{aligned}
\left\langle-\Delta \phi_{\varepsilon}\right. & \left.-(p+\varepsilon) K\left(\varepsilon^{1 /(N-2)}|y|\right) W_{r, \Lambda}^{p-1+\varepsilon} \phi_{\varepsilon}, Z_{1, \ell}\right\rangle \\
& =\left\langle-\Delta Z_{1, \ell}-(p+\varepsilon) K\left(\varepsilon^{1 /(N-2)}|y|\right) W_{r, \Lambda}^{p-1+\varepsilon} Z_{1, \ell}, \phi_{\varepsilon}\right\rangle
\end{aligned}
$$




$$
\begin{aligned}
& \begin{array}{l}
=\left\langle-\Delta Z_{1, \ell}-p K\left(\varepsilon^{1 /(N-2)}|y|\right) W_{r, \Lambda}^{p-1} Z_{1, \ell}, \phi_{\varepsilon}\right\rangle \\
+p\left\langle K\left(\varepsilon^{1 /(N-2)}|y|\right)\left(W_{r, \Lambda}^{p-1}-W_{r, \Lambda}^{p-1+\varepsilon}\right) Z_{1, \ell}, \phi_{\varepsilon}\right\rangle \\
\left.\quad-\varepsilon\left\langle K\left(\varepsilon^{1 /(N-2)}|y|\right) W_{r, \Lambda}^{p-1+\varepsilon}\right) Z_{1, \ell}, \phi_{\varepsilon}\right\rangle
\end{array} \\
& =o\left(\left\|\phi_{\varepsilon}\right\|_{*}\right) .
\end{aligned}
$$

However, there is a constant $c^{\prime}>0$ such that

$$
\sum_{i=1}^{k}\left\langle U_{x_{i}, \Lambda}^{p-1} Z_{i, t}, Z_{1, \ell}\right\rangle=\left(c^{\prime}+o(1)\right) \delta_{t \ell} .
$$

Hence we find from (2-4) that

$$
c_{\ell}=o\left(\left\|\phi_{\varepsilon}\right\|_{*}\right)+O\left(\left\|h_{\varepsilon}\right\|_{* *}\right) .
$$

Therefore,

$$
\left\|\phi_{\varepsilon}\right\|_{*} \leq o(1)+\left\|h_{\varepsilon}\right\|_{* *}+\frac{\sum_{j=1}^{k} \frac{1}{\left(1+\left|y-x_{j}\right|\right)^{\frac{1}{2}(N-2)+\tau+\vartheta}}}{\sum_{j=1}^{k} \frac{1}{\left(1+\left|y-x_{j}\right|\right)^{\frac{1}{2}(N-2)+\tau}} .}
$$

Noting that $\left\|\phi_{\varepsilon}\right\|_{*}=1$, we obtain from (2-5) that there is $R>0$ such that

$$
\left\|\phi_{\varepsilon}(y)\right\|_{L^{\infty}\left(B_{R}\left(x_{i}\right)\right)} \geq a>0 \text { for some } i \text {. }
$$

Furthermore, for this particular $i$, the translated version $\bar{\phi}_{\varepsilon}(y)=\phi_{\varepsilon}\left(y-x_{i}\right)$ converges uniformly on any compact set to a solution $u$ of

$$
-\Delta u-p U_{0, \Lambda}^{p-1} u=0 \quad \text { in } \mathbb{R}^{N}
$$

for some $\Lambda \in\left[L_{0}, L_{1}\right]$. Since $u$ is perpendicular to the kernel of (2-7), we have $u \equiv 0$, which contradicts $\|u(y)\|_{L^{\infty}\left(B_{R}\left(x_{i}\right)\right)} \geq a>0$.

The following proposition is a direct consequence of combining Proposition 4.1 in [del Pino et al. 2003] with Lemma 2.1.

Proposition 2.2. There exists $\varepsilon_{0}>0$ and a constant $C>0$ such that for all $\varepsilon \leq \varepsilon_{0}$ and all $h_{\varepsilon} \in L_{* *}^{\infty}$, problem (2-3) has a unique solution $\phi_{\varepsilon} \equiv \mathcal{L}_{\varepsilon}\left(h_{\varepsilon}\right) \in L_{*}^{\infty}$. Moreover,

$$
\left\|\mathcal{L}_{\varepsilon}\left(h_{\varepsilon}\right)\right\|_{*} \leq C\left\|h_{\varepsilon}\right\|_{* *}, \quad\left|c_{\ell}\right| \leq C\left\|h_{\varepsilon}\right\|_{* *} .
$$


In order to prove the main theorem, we will prove that problem (1-4) admits a solution of the form $u=W_{r, \Lambda}+\phi$, where $W_{r, \Lambda}=\sum_{j=1}^{k} P U_{x_{j}, \Lambda}$ and $\phi \in \mathcal{H}_{s}$ is small and satisfies $\left\langle U_{x_{i}, \Lambda}^{p-1} Z_{i, l}, \phi\right\rangle=0, i=1,2, \ldots, k, l=1,2$.

We consider the perturbation problem

$$
\left\{\begin{array}{l}
-\Delta\left(W_{r, \Lambda}+\phi\right)=K\left(\varepsilon^{\frac{1}{N-2}}|y|\right)\left(W_{r, \Lambda}+\phi\right)^{p+\varepsilon}+\sum_{\ell=1}^{2} c_{\ell} \sum_{i=1}^{k} U_{x_{i}, \Lambda}^{p-1} Z_{i, \ell} \text { in } B_{*}(0), \\
\phi \in \mathcal{H}_{s}, \quad\left\langle\sum_{i=1}^{k} U_{x_{i}, \Lambda}^{p-1} Z_{i, \ell}, \phi\right\rangle=0, \quad \ell=1,2 .
\end{array}\right.
$$

Proposition 2.3. There is $\varepsilon_{0}>0$ such that for any $\varepsilon \leq \varepsilon_{0}$, any $\Lambda \in\left[L_{0}, L_{1}\right]$, and

$$
r \in\left[\varepsilon^{-\frac{1}{N-2}}\left(1-r_{0} \varepsilon^{\frac{1}{N-1}}\right), \varepsilon^{-\frac{1}{N-2}}\left(1-r_{1} \varepsilon^{\frac{1}{N-1}}\right)\right],
$$

problem (2-9) has a unique solution $\phi=\phi_{r, \Lambda}$ satisfying

$$
\|\phi\|_{*} \leq C \varepsilon^{\left(\frac{1}{2}+\sigma\right) /(N-2)}, \quad\left|c_{\ell}\right| \leq C \varepsilon^{\left(\frac{1}{2}+\sigma\right) /(N-2)}
$$

where $\sigma>0$ is a small constant.

Let

$$
\begin{aligned}
N_{\varepsilon}(\phi) & =K\left(\varepsilon^{\frac{1}{N-2}}|y|\right)\left(\left(W_{r, \Lambda}+\phi\right)^{p+\varepsilon}-W_{r, \Lambda}^{p+\varepsilon}-(p+\varepsilon) W_{r, \Lambda}^{p-1+\varepsilon} \phi\right), \\
l_{\varepsilon} & =K\left(\varepsilon^{\frac{1}{N-2}}|y|\right) W_{r, \Lambda}^{p+\varepsilon}-\sum_{j=1}^{k} U_{x_{j}, \Lambda}^{p} .
\end{aligned}
$$

Then problem (2-9) can be written as

$$
\left\{\begin{array}{l}
-\Delta \phi-(p+\varepsilon) K\left(\varepsilon^{\frac{1}{N-2}}|y|\right) W_{r, \Lambda}^{p-1+\varepsilon} \phi \\
=N_{\varepsilon}(\phi)+l_{\varepsilon}+\sum_{\ell=1}^{2} c_{\ell} \sum_{i=1}^{k} U_{x_{i}, \Lambda}^{p-1} Z_{i, \ell} \quad \text { in } B_{*}(0), \\
\phi \in \mathcal{H}_{s}, \quad\left\langle\sum_{i=1}^{k} U_{x_{i}, \Lambda}^{p-1} Z_{i, \ell}, \phi\right\rangle=0, \quad \ell=1,2 .
\end{array}\right.
$$

We will use the contraction mapping theorem to prove that problem (2-9) is uniquely solvable under the condition that $\|\phi\|_{*}$ is small enough. So we need to estimate $N_{\varepsilon}(\phi)$ and $l_{\varepsilon}$.

Lemma 2.4. If $N \geq 4$, then

$$
\left\|N_{\varepsilon}(\phi)\right\|_{* *} \leq C\|\phi\|_{*}^{\min \{p+\varepsilon, 2\}} .
$$

Proof. We have

$$
\left|N_{\varepsilon}(\phi)\right| \leq \begin{cases}C|\phi|^{p+\varepsilon}, & N \geq 7 \\ C\left(W_{r, \Lambda}^{p-2+\varepsilon} \phi^{2}+|\phi|^{p+\varepsilon}\right), & N=4,5,6 .\end{cases}
$$


Firstly, we consider $N \geq 7$. By the Hölder inequality, we have

$$
\begin{aligned}
\left|N_{\varepsilon}(\phi)\right| \leq C\|\phi\|_{*}^{p+\varepsilon} & \left(\sum_{j=1}^{k} \frac{1}{\left(1+\left|y-x_{j}\right|\right)^{\frac{1}{2}(N-2)+\tau}}\right)^{p+\varepsilon} \\
\leq C\|\phi\|_{*}^{p+\varepsilon} & \left(\sum_{j=1}^{k} \frac{1}{\left(1+\left|y-x_{j}\right|\right)^{\frac{1}{2}(N+2)+\tau}}\right) \\
& \times\left(\sum_{j=1}^{k} \frac{1}{\left(1+\left|y-x_{j}\right|\right)^{\frac{p+\varepsilon}{p+\varepsilon-1}\left(\frac{1}{2}(N-2)+\tau\right)-\frac{1}{p+\varepsilon-1}\left(\frac{1}{2}(N+2)+\tau\right)}}\right)^{p+\varepsilon-1} \\
\leq C\|\phi\|_{*}^{p+\varepsilon} & \left(\sum_{j=1}^{k} \frac{1}{\left(1+\left|y-x_{j}\right|\right)^{\frac{1}{2}(N+2)+\tau}}\right) .
\end{aligned}
$$

Thus, the result follows.

Suppose that $N=4,5,6$. Using the fact that $N-2>\frac{1}{2}(N-2)+\tau$, we get

$$
\begin{aligned}
\left|N_{\varepsilon}(\phi)\right| & \leq C\|\phi\|_{*}^{2}\left(\sum_{j=1}^{k} \frac{1}{\left(1+\left|y-x_{j}\right|\right)^{N-2}}\right)^{p-2+\varepsilon}\left(\sum_{j=1}^{k} \frac{1}{\left(1+\left|y-x_{j}\right|\right)^{\frac{1}{2}(N-2)+\tau}}\right)^{2} \\
& +C\|\phi\|_{*}^{p+\varepsilon}\left(\sum_{j=1}^{k} \frac{1}{\left(1+\left|y-x_{j}\right|\right)^{\frac{1}{2}(N+2)+\tau}}\right) \\
& \leq C\|\phi\|_{*}^{2}\left(\sum_{j=1}^{k} \frac{1}{\left(1+\left|y-x_{j}\right|\right)^{\frac{1}{2}(N-2)+\tau}}\right)^{p+\varepsilon}+C\|\phi\|_{*}^{p+\varepsilon}\left(\sum_{j=1}^{k} \frac{1}{\left(1+\left|y-x_{j}\right|\right)^{\frac{1}{2}(N+2)+\tau}}\right) \\
& \leq C\|\phi\|_{*}^{2}\left(\sum_{j=1}^{k} \frac{1}{\left(1+\left|y-x_{j}\right|\right)^{\frac{1}{2}(N+2)+\tau}}\right) .
\end{aligned}
$$

So we have proved that for $N \geq 4$,

$$
\left\|N_{\varepsilon}(\phi)\right\|_{* *} \leq C\|\phi\|_{*}^{\min \{p+\varepsilon, 2\}} .
$$

Lemma 2.5. Assume that $r \in\left[\varepsilon^{-\frac{1}{N-2}}\left(1-r_{0} \varepsilon^{\frac{1}{N-1}}\right), \varepsilon^{-\frac{1}{N-2}}\left(1-r_{1} \varepsilon^{\frac{1}{N-1}}\right)\right]$. If $N \geq 4$, then

$$
\left\|l_{\varepsilon}\right\|_{* *} \leq C \varepsilon^{\left(\frac{1}{2}+\sigma\right) /(N-2)} .
$$

Proof. Define

$$
\Omega_{j}=\left\{y: y=\left(y^{\prime}, y^{\prime \prime}\right) \in B_{\varepsilon^{-1 /(N-2)}}(0),\left\langle\frac{y^{\prime}}{\left|y^{\prime}\right|}, \frac{x_{j}}{\left|x_{j}\right|}\right\rangle \geq \cos \frac{\pi}{k}\right\} .
$$


We have

$$
\begin{aligned}
l_{\varepsilon}= & K\left(\varepsilon^{1 /(N-2)}|y|\right)\left(W_{r, \Lambda}^{p+\varepsilon}-W_{r, \Lambda}^{p}\right)+K\left(\varepsilon^{1 /(N-2)}|y|\right)\left(W_{r, \Lambda}^{p}-\sum_{j=1}^{k} P U_{x_{j}, \Lambda}^{p}\right) \\
& +K\left(\varepsilon^{1 /(N-2)}|y|\right)\left(\sum_{j=1}^{k} P U_{x_{j}, \Lambda}^{p}-\sum_{j=1}^{k} U_{x_{j}, \Lambda}^{p}\right)+\sum_{j=1}^{k} U_{x_{j}, \Lambda}^{p}\left(K\left(\varepsilon^{1 /(N-2)}|y|\right)-1\right) \\
= & : J_{0}+J_{1}+J_{2}+J_{3} .
\end{aligned}
$$

Estimate of $J_{0}$.

$$
\begin{aligned}
\left|J_{0}\right| & \leq C \varepsilon W_{r, \Lambda}^{p}\left|\ln W_{r, \Lambda}\right| \\
& \leq C \varepsilon\left(\sum_{j=1}^{k} \frac{1}{\left(1+\left|y-x_{j}\right|\right)^{N-2}}\right)^{p} \ln \sum_{j=1}^{k} \frac{1}{\left(1+\left|y-x_{j}\right|\right)^{N-2}} \\
& \leq C \varepsilon\left(\sum_{j=1}^{k} \frac{1}{\left(1+\left|y-x_{j}\right|\right)^{\frac{1}{2}(N+2)+\tau}}\right)\left(\sum_{j=1}^{k} \frac{1}{\left(1+\left|y-x_{j}\right|\right)^{\frac{N+2}{4}\left(\frac{N-2}{2}-\frac{N-2}{N+2} \tau\right)}}\right)^{\frac{4}{N-2}} \\
& \leq C \varepsilon \sum_{j=1}^{k} \frac{1}{\left(1+\left|y-x_{j}\right|\right)^{\frac{1}{2}(N+2)+\tau}} .
\end{aligned}
$$

Estimate of $J_{1}$. From the symmetry, we can assume that $y \in \Omega_{1}$. Then,

$$
\left|y-x_{j}\right| \geq\left|y-x_{1}\right|, \quad y \in \Omega_{1}, j \neq 1 \text {. }
$$

Firstly, we claim

$$
\frac{1}{1+\left|y-x_{j}\right|} \leq \frac{C}{\left|x_{j}-x_{1}\right|}, \quad y \in \Omega_{1}, j \neq 1
$$

In fact, if $\left|y-x_{1}\right| \leq \frac{1}{2}\left|x_{1}-x_{j}\right|$, then $\left|y-x_{j}\right| \geq \frac{1}{2}\left|x_{j}-x_{1}\right|$. If $\left|y-x_{1}\right| \geq \frac{1}{2}\left|x_{j}-x_{1}\right|$, then $\left|y-x_{j}\right| \geq\left|y-x_{1}\right| \geq \frac{1}{2}\left|x_{j}-x_{1}\right|$.

It's easy to verify that

$$
\left|J_{1}\right| \leq C \frac{1}{\left(1+\left|y-x_{1}\right|\right)^{4}} \sum_{j=2}^{k} \frac{1}{\left(1+\left|y-x_{j}\right|\right)^{N-2}}+C\left(\sum_{j=2}^{k} \frac{1}{\left(1+\left|y-x_{j}\right|\right)^{N-2}}\right)^{p} .
$$

Using (2-11), taking $1<\varrho \leq N-2$, we obtain for $y \in \Omega_{1}$,

$$
\frac{1}{\left(1+\left|y-x_{1}\right|\right)^{4}} \frac{1}{\left(1+\left|y-x_{j}\right|\right)^{N-2}} \leq C \frac{1}{\left(1+\left|y-x_{1}\right|\right)^{N+2-\varrho}} \frac{1}{\left|x_{j}-x_{1}\right|^{\varrho}}, \quad j \neq 1 .
$$


Take $\varrho>\max \left\{\frac{1}{2}(N-1), 1\right\}$ satisfying $N+2-\varrho \geq \frac{1}{2}(N+2)+\tau$. Then

$$
\begin{aligned}
& \frac{1}{\left(1+\left|y-x_{1}\right|\right)^{4}} \sum_{j=2}^{k} \frac{1}{\left(1+\left|y-x_{j}\right|\right)^{N-2}} \leq \frac{C}{\left(1+\left|y-x_{1}\right|\right)^{N+2-\varrho}}\left(k \varepsilon^{1 /(N-2)}\right)^{\varrho} \\
& =\frac{C}{\left(1+\left|y-x_{1}\right|\right)^{N+2-\varrho}} \varepsilon^{\varrho /((N-1)(N-2))} \leq \frac{C}{\left(1+\left|y-x_{1}\right|\right)^{\frac{1}{2}(N+2)+\tau}} \varepsilon^{\left(\frac{1}{2}+\sigma\right) /(N-2)} .
\end{aligned}
$$

By the Hölder inequality, we find

$$
\begin{aligned}
& \left(\sum_{j=2}^{k} \frac{1}{\left(1+\left|y-x_{j}\right|\right)^{N-2}}\right)^{p} \\
& \quad \leq \sum_{j=2}^{k} \frac{1}{\left(1+\left|y-x_{j}\right|\right)^{\frac{1}{2}(N+2)+\tau}}\left(\sum_{j=2}^{k} \frac{1}{\left(1+\left|y-x_{j}\right|\right)^{\frac{N+2}{4}\left(\frac{N-2}{2}-\tau \frac{N-2}{N+2}\right)}}\right)^{\frac{4}{N-2}} .
\end{aligned}
$$

Noticing that $\frac{N+2}{N-2}\left(\frac{N-2}{2}-\tau \frac{N-2}{N+2}\right)>\frac{N-1}{2}$ if $N \geq 4$, we deduce that

$$
\begin{aligned}
& \left(\sum_{j=2}^{k} \frac{1}{\left(1+\left|y-x_{j}\right|\right)^{N-2}}\right)^{p} \\
& \leq C\left(\sum_{j=2}^{k} \frac{1}{\left|x_{j}-x_{1}\right|^{\frac{N+2}{4}\left(\frac{N-2}{2}-\tau \frac{N-2}{N+2}\right)}}\right)^{\frac{4}{N-2}} \sum_{j=1}^{k} \frac{1}{\left(1+\left|y-x_{j}\right|\right)^{\frac{1}{2}(N+2)+\tau}} \\
& \leq C\left(k \varepsilon^{1 /(N-2)}\right)^{\frac{N+2}{N-2}\left(\frac{N-2}{2}-\tau \frac{N-2}{N+2}\right)} \sum_{j=1}^{k} \frac{1}{\left(1+\left|y-x_{j}\right|\right)^{\frac{1}{2}(N+2)+\tau}} \\
& \leq C \varepsilon^{\left(\frac{1}{2}+\sigma\right) /(N-2)} \sum_{j=1}^{k} \frac{1}{\left(1+\left|y-x_{j}\right|\right)^{\frac{1}{2}(N+2)+\tau}} \text {. }
\end{aligned}
$$

Hence, we conclude that if $N \geq 4$,

$$
\left\|J_{1}\right\|_{* *} \leq C \varepsilon^{\left(\frac{1}{2}+\sigma\right) /(N-2)} .
$$

Estimate of $J_{2}$. Let $H(y, x)$ be the regular part of the Green function for $-\Delta$ in

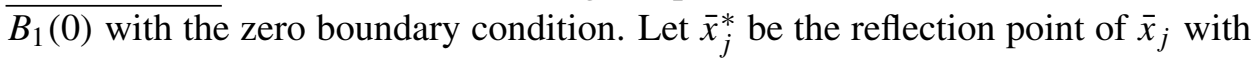
respect to $\partial B_{1}(0)$. Then

$$
\varepsilon H\left(\bar{y}, \bar{x}_{j}\right)=\frac{C \varepsilon}{\left|\bar{y}-\bar{x}_{j}^{*}\right|^{N-2}} \leq \frac{C}{\left(1+\left|y-x_{j}\right|\right)^{N-2}} .
$$


By direct calculation, we have

$$
\begin{aligned}
\left|J_{2}\right| & \leq C \sum_{j=1}^{k} \frac{C \varepsilon}{\left(1+\left|y-x_{j}\right|\right)^{4}} H\left(\bar{y}, \bar{x}_{j}\right) \\
& \leq \sum_{j=1}^{k} \frac{C}{\left(1+\left|y-x_{j}\right|\right)^{4+(1-\gamma)(N-2)}}\left(\varepsilon H\left(\bar{y}, \bar{x}_{j}\right)\right)^{\gamma} \\
& \leq C \varepsilon^{\gamma /(N-1)} \sum_{j=1}^{k} \frac{C}{\left(1+\left|y-x_{j}\right|\right)^{4+(1-\gamma)(N-2)}} \\
& \leq C \varepsilon^{\left(\frac{1}{2}+\sigma\right) /(N-2)} \sum_{j=1}^{k} \frac{C}{\left(1+\left|y-x_{j}\right|\right)^{\frac{1}{2}(N+2)+\tau}},
\end{aligned}
$$

where $\gamma>0$ satisfies $\gamma(N-2) /(N-1)>\frac{1}{2}$ and $4+(1-\gamma)(N-2) \geq \frac{1}{2}(N+2)+\tau$.

Estimate of $J_{3}$. For $y \in \Omega_{1}$ and $j>1$, using (2-11), we find

$$
U_{x_{j}, \Lambda}^{p} \leq \frac{C}{\left(1+\left|y-x_{1}\right|\right)^{\frac{1}{2}(N+2)+\tau}} \frac{1}{\left|x_{j}-x_{1}\right|^{\frac{1}{2}(N+2)-\tau}} .
$$

Thus, we have

$$
\begin{aligned}
\left|\sum_{j=2}^{k}\left(K\left(\varepsilon^{1 /(N-2)}|y|\right)-1\right) U_{x_{j}, \Lambda}^{p}\right| & \leq \frac{C}{\left(1+\left|y-x_{1}\right|\right)^{\frac{1}{2}(N+2)+\tau}} \sum_{j=2}^{k} \frac{1}{\left|x_{j}-x_{1}\right|^{\frac{1}{2}(N+2)-\tau}} \\
& \leq \frac{C}{\left(1+\left|y-x_{1}\right|\right)^{\frac{1}{2}(N+2)+\tau}}\left(k \varepsilon^{1 /(N-2)}\right)^{\frac{1}{2}(N+2)-\tau} \\
& \leq \frac{C}{\left(1+\left|y-x_{1}\right|\right)^{\frac{1}{2}(N+2)+\tau}} \varepsilon^{\left(\frac{1}{2}+\sigma\right) /(N-2)} .
\end{aligned}
$$

If $y \in \Omega_{1}$ and ||$y\left|-\varepsilon^{-1 /(N-2)}\right| \geq \delta \varepsilon^{-1 /(N-2)}$, where $\delta>0$ is a fixed constant, then

$$
|| y|-| x_{1}|| \geq|| y\left|-\varepsilon^{-1 /(N-2)}\right|-|| x_{1}\left|-\varepsilon^{-1 /(N-2)}\right| \geq \frac{1}{2} \delta \varepsilon^{-1 /(N-2)} .
$$

So, we obtain

$$
\left|U_{x_{1}, \Lambda}^{p}\left(K\left(\varepsilon^{1 /(N-2)}|y|\right)-1\right)\right| \leq \frac{C}{\left(1+\left|y-x_{1}\right|\right)^{\frac{1}{2}(N+2)+\tau}} \varepsilon^{\left(\frac{1}{2}(N+2)-\tau\right) /(N-2)} .
$$


If $y \in \Omega_{1}$ and ||$y\left|-\varepsilon^{-1 /(N-2)}\right| \leq \delta \varepsilon^{-1 /(N-2)}$, then

$$
\begin{aligned}
\left|K\left(\varepsilon^{1 /(N-2)}|y|\right)-1\right| & \leq C\left|\varepsilon^{1 /(N-2)}\right| y|-1| \\
& \leq C \varepsilon^{1 /(N-2)}\left(|| y|-| x_{1}||+|| x_{1}\left|-\varepsilon^{-1 /(N-2)}\right|\right) \\
& \leq C \varepsilon^{1 /(N-2)}|| y|-| x_{1}||+C \varepsilon^{1 /(N-1)} \\
& \leq C \varepsilon^{1 /(N-2)}|| y|-| x_{1}||+C \varepsilon^{\left(\frac{1}{2}+\sigma\right) /(N-2)},
\end{aligned}
$$

and

$$
|| y|-| x_{1}|| \leq|y|\left|-\varepsilon^{-1 /(N-2)}\right|+|| x_{1}\left|-\varepsilon^{-1 /(N-2)}\right| \leq 2 \delta \varepsilon^{-1 /(N-2)} .
$$

Since

$$
\begin{aligned}
\frac{\varepsilon^{1 /(N-2)}|| y|-| x_{1}||}{\left(1+\left|y-x_{1}\right|\right)^{N+2}} & \leq C \varepsilon^{\left(\frac{1}{2}+\sigma\right) /(N-2)} \frac{|| y|-| x_{1}||^{\frac{1}{2}+\sigma}}{\left(1+\left|y-x_{1}\right|\right)^{N+2}} \\
& \leq \frac{C \varepsilon^{\left(\frac{1}{2}+\sigma\right) /(N-2)}}{\left(1+\left|y-x_{1}\right|\right)^{N+\frac{3}{2}-\sigma}} \leq \frac{C \varepsilon^{\left(\frac{1}{2}+\sigma\right) /(N-2)}}{\left(1+\left|y-x_{1}\right|\right)^{\frac{1}{2}(N+2)+\tau}},
\end{aligned}
$$

we get

$$
\left|U_{x_{1}, \Lambda}^{p}\left(K\left(\varepsilon^{1 /(N-2)}|y|\right)-1\right)\right| \leq \frac{C \varepsilon^{\left(\frac{1}{2}+\sigma\right) /(N-2)}}{\left(1+\left|y-x_{1}\right|\right)^{\frac{1}{2}(N+2)+\tau}} .
$$

As a result, we deduce

$$
\left\|J_{3}\right\|_{* *} \leq C \varepsilon^{\left(\frac{1}{2}+\sigma\right) /(N-2)} .
$$

Proof of Proposition 2.3. Recall that

$$
k=\left[\varepsilon^{-1 /(N-1)}\right], \quad N \geq 4 .
$$

Let

$$
\begin{aligned}
& E=\left\{u \in \mathcal{H}_{S} \cap L_{*}^{\infty}:\|u\|_{*} \leq \varepsilon^{1 /(2(N-2))}\right. \text { and } \\
& \left.\qquad \int_{B_{*}(0)} U_{x_{i}, \Lambda}^{p-1} Z_{i, \ell} u=0, i=1, \ldots, k, \ell=1,2\right\} .
\end{aligned}
$$

Then, (2-10) is equivalent to

$$
\phi=A_{\varepsilon}(\phi)=: \mathcal{L}_{\varepsilon}\left(N_{\varepsilon}(\phi)\right)+\mathcal{L}_{\varepsilon}\left(l_{\varepsilon}\right),
$$

where $\mathcal{L}_{\varepsilon}$ is defined in Proposition 2.2. We will prove that $A_{\varepsilon}$ is a contraction map from $E$ to $E$. First, $A_{\varepsilon}(E) \subset E$ because

$$
\begin{aligned}
\left\|A_{\varepsilon}(\phi)\right\|_{*} & \leq C\left\|N_{\varepsilon}(\phi)\right\|_{* *}+C\left\|l_{\varepsilon}\right\|_{* *} \\
& \leq C\|\phi\|_{*}^{\min \{p+\varepsilon, 2\}}+C\left\|l_{\varepsilon}\right\|_{* *} \leq C \varepsilon^{\left(\frac{1}{2}+\sigma\right) /(N-2)} \leq \varepsilon^{1 /(2(N-2))} .
\end{aligned}
$$


Next we write

$$
\left\|A_{\varepsilon}\left(\phi_{1}\right)-A_{\varepsilon}\left(\phi_{2}\right)\right\|_{*}=\left\|\mathcal{L}_{\varepsilon}\left(N_{\varepsilon}\left(\phi_{1}\right)\right)-\mathcal{L}_{\varepsilon}\left(N_{\varepsilon}\left(\phi_{2}\right)\right)\right\|_{*} \leq C\left\|N_{\varepsilon}\left(\phi_{1}\right)-N_{\varepsilon}\left(\phi_{2}\right)\right\|_{* *} .
$$

If $N \geq 7$, then

$$
\left|N_{\varepsilon}^{\prime}(t)\right| \leq C|t|^{p-1+\varepsilon}
$$

Thus, we have

$$
\begin{aligned}
& \left|N_{\varepsilon}\left(\phi_{1}\right)-N_{\varepsilon}\left(\phi_{2}\right)\right| \\
& \quad \leq C\left(\left|\phi_{1}\right|^{p-1+\varepsilon}+\left|\phi_{2}\right|^{p-1+\varepsilon}\right)\left|\phi_{1}-\phi_{2}\right| \\
& \quad \leq C\left(\left\|\phi_{1}\right\|_{*}^{p-1+\varepsilon}+\left\|\phi_{2}\right\|_{*}^{p-1+\varepsilon}\right)\left\|\phi_{1}-\phi_{2}\right\|_{*}\left(\sum_{j=1}^{k} \frac{1}{\left(1+\left|y-x_{j}\right|\right)^{\frac{1}{2}(N-2)+\tau}}\right)^{p+\varepsilon} \\
& \quad \leq C\left(\left\|\phi_{1}\right\|_{*}^{p-1+\varepsilon}+\left\|\phi_{2}\right\|_{*}^{p-1+\varepsilon}\right)\left\|\phi_{1}-\phi_{2}\right\|_{*} \sum_{j=1}^{k} \frac{1}{\left(1+\left|y-x_{j}\right|\right)^{\frac{1}{2}(N+2)+\tau}} .
\end{aligned}
$$

As a consequence,

$$
\begin{aligned}
\left\|A_{\varepsilon}\left(\phi_{1}\right)-A_{\varepsilon}\left(\phi_{2}\right)\right\|_{*} & \leq C\left\|N_{\varepsilon}\left(\phi_{1}\right)-N_{\varepsilon}\left(\phi_{2}\right)\right\|_{* *} \\
& \leq C\left(\left\|\phi_{1}\right\|_{*}^{p-1+\varepsilon}+\left\|\phi_{2}\right\|_{*}^{p-1+\varepsilon}\right)\left\|\phi_{1}-\phi_{2}\right\|_{*} \leq \frac{1}{2}\left\|\phi_{1}-\phi_{2}\right\|_{*} .
\end{aligned}
$$

For $N=4,5,6$,

$$
\left|N_{\varepsilon}^{\prime}(t)\right| \leq C W_{r, \Lambda}^{p-2+\varepsilon}|t|+C|t|^{p-1+\varepsilon} .
$$

So we have

$$
\begin{aligned}
& \left|N_{\varepsilon}\left(\phi_{1}\right)-N_{\varepsilon}\left(\phi_{2}\right)\right| \\
& \leq C\left(\left|\phi_{1}\right|^{p-1+\varepsilon}+\left|\phi_{2}\right|^{p-1+\varepsilon}\right)\left|\phi_{1}-\phi_{2}\right|+C\left(\left|\phi_{1}\right|+\left|\phi_{2}\right|\right) W_{r, \Lambda}^{p-2+\varepsilon}\left|\phi_{1}-\phi_{2}\right| \\
& \leq C\left(\left\|\phi_{1}\right\|_{*}^{p-1+\varepsilon}+\left\|\phi_{2}\right\|_{*}^{p-1+\varepsilon}\right)\left\|\phi_{1}-\phi_{2}\right\|_{*}\left(\sum_{j=1}^{k} \frac{1}{\left(1+\left|y-x_{j}\right|\right)^{\frac{1}{2}(N-2)+\tau}}\right)^{p+\varepsilon} \\
& \quad+C\left(\left\|\phi_{1}\right\|_{*}+\left\|\phi_{2}\right\|_{*}\right)\left\|\phi_{1}-\phi_{2}\right\|_{*} W_{r, \Lambda}^{p-2+\varepsilon}\left(\sum_{j=1}^{k} \frac{1}{\left(1+\left|y-x_{j}\right|\right)^{\frac{1}{2}(N-2)+\tau}}\right)^{2} \\
& \quad C\left(\left\|\phi_{1}\right\|_{*}+\left\|\phi_{2}\right\|_{*}\right)\left\|\phi_{1}-\phi_{2}\right\|_{*} \sum_{j=1}^{k} \frac{1}{\left(1+\left|y-x_{j}\right|\right)^{\frac{1}{2}(N+2)+\tau}} .
\end{aligned}
$$

In either case, we see that $A_{\varepsilon}$ is a contraction map. By the contraction mapping theorem, there is a unique $\phi \in E$ such that

$$
\phi=A_{\varepsilon}(\phi) .
$$


Moreover, it follows from Proposition 2.2 that

$$
\|\phi\|_{*} \leq C\left\|l_{\varepsilon}\right\|_{* *}+C\left\|N_{\varepsilon}(\phi)\right\|_{* *} \leq C\left\|l_{\varepsilon}\right\|_{* *}+C\|\phi\|_{*}^{\min \{p+\varepsilon, 2\}},
$$

which implies

$$
\|\phi\|_{*} \leq C \varepsilon^{\left(\frac{1}{2}+\sigma\right) /(N-2)}, \quad\left|c_{\ell}\right| \leq C \varepsilon^{\left(\frac{1}{2}+\sigma\right) /(N-2)} .
$$

\section{Proof of the main results}

In this section, we will choose $(r, \Lambda)$ such that $W_{r, \Lambda}+\phi_{r, \Lambda}$ is a solution of (1-4), where $\phi_{r, \Lambda}$ is the map obtained in Proposition 2.3.

Lemma 3.1. If $(r, \Lambda)$ satisfies

$\int_{B_{*}(0)}\left(\nabla\left(W_{r, \Lambda}+\phi_{r, \Lambda}\right) \nabla \frac{\partial W_{r, \Lambda}}{\partial r}-K\left(\varepsilon^{1 /(N-2)}|y|\right)\left(W_{r, \Lambda}+\phi_{r, \Lambda}\right)^{p+\varepsilon} \frac{\partial W_{r, \Lambda}}{\partial r}\right)=0$,

$\int_{B_{*}(0)}\left(\nabla\left(W_{r, \Lambda}+\phi_{r, \Lambda}\right) \nabla \frac{\partial W_{r, \Lambda}}{\partial \Lambda}-K\left(\varepsilon^{1 /(N-2)}|y|\right)\left(W_{r, \Lambda}+\phi_{r, \Lambda}\right)^{p+\varepsilon} \frac{\partial W_{r, \Lambda}}{\partial \Lambda}\right)=0$,

then $W_{r, \Lambda}+\phi_{r, \Lambda}$ is a solution of (1-4).

Proof. It follows from Proposition 2.3 that if (3-1) and (3-2) hold, then by symmetry,

$$
c_{1}\left\langle U_{x_{1}, \Lambda}^{p-1} \frac{\partial P U_{x_{1}, \Lambda}}{\partial r}, \frac{\partial W_{r, \Lambda}}{\partial r}\right\rangle=0=c_{2}\left\langle U_{x_{1}, \Lambda}^{p-1} \frac{\partial P U_{x_{1}, \Lambda}}{\partial \Lambda}, \frac{\partial W_{r, \Lambda}}{\partial \Lambda}\right\rangle,
$$

which implies that $c_{1}=c_{2}=0$. Hence $W_{r, \Lambda}+\phi_{r, \Lambda}$ is a solution of (1-4).

In the rest of this section, we need to solve (3-1) and (3-2).

Proposition 3.2. Equations (3-1) and (3-2) are equivalent to

$$
-\frac{\varepsilon H\left(\bar{x}_{1}, \bar{x}_{1}\right)}{\Lambda^{N-1}}+\sum_{i=2}^{k} \frac{\varepsilon G\left(\bar{x}_{i}, \bar{x}_{1}\right)}{\Lambda^{N-1}}+O\left(\varepsilon^{(1+\sigma) /(N-2)}\right)=0
$$

and

(3-5) $\frac{B_{2} \varepsilon}{\Lambda^{N-2}} \frac{\partial H\left(\bar{x}_{1}, \bar{x}_{1}\right)}{\partial d}+B_{3} K^{\prime}(1)+\sum_{i=2}^{k} \frac{B_{2} \varepsilon}{\Lambda^{N-2}} \frac{\partial G\left(\bar{x}_{i}, \bar{x}_{1}\right)}{\partial d}+O\left(\varepsilon^{\sigma /(N-2)}\right)=0$,

respectively, where $d=1-\varepsilon^{1 /(N-2)} r, B_{1}, B_{2}$ and $B_{3}$ are the same positive constants as in Proposition A.1 and $\sigma>0$ is a small constant.

Proof. Here we prove only the first one. The second can be proved similarly by noting that $\partial / \partial d=-\varepsilon^{-1 /(N-2)} \partial / \partial r$. 
First, we see that

$$
\int_{B_{*}(0)} \nabla\left(W_{r, \Lambda}+\phi_{r, \Lambda}\right) \nabla \frac{\partial W_{r, \Lambda}}{\partial \Lambda}=\int_{B_{*}(0)} \nabla W_{r, \Lambda} \nabla \frac{\partial W_{r, \Lambda}}{\partial \Lambda},
$$

and

$$
\begin{aligned}
\int_{B_{*}(0)} K\left(\varepsilon^{1 /(N-2)}|y|\right)\left(W_{r, \Lambda}+\phi_{r, \Lambda}\right)^{p+\varepsilon} \frac{\partial W_{r, \Lambda}}{\partial \Lambda} & \\
=\int_{B_{*}(0)} K\left(\varepsilon^{1 /(N-2)}|y|\right) W_{r, \Lambda}^{p+\varepsilon} \frac{\partial W_{r, \Lambda}}{\partial \Lambda} & \\
& +(p+\varepsilon) \int_{B_{*}(0)} K\left(\varepsilon^{1 /(N-2)}|y|\right) W_{r, \Lambda}^{p-1+\varepsilon} \frac{\partial W_{r, \Lambda}}{\partial \Lambda} \phi_{r, \Lambda} \\
& +O\left(\int_{B_{*}(0)} W_{r, \Lambda}^{p-1+\varepsilon}\left|\phi_{r, \Lambda}\right|^{2}\right) .
\end{aligned}
$$

On the other hand, noticing that $\phi_{r, \Lambda} \in E$, we have

$$
\begin{aligned}
\int_{B_{*}(0)} K\left(\varepsilon^{1 /(N-2)}|y|\right) W_{r, \Lambda}^{p-1+\varepsilon} \frac{\partial W_{r, \Lambda}}{\partial \Lambda} \phi_{r, \Lambda} & \\
= & \int_{B_{*}(0)} K\left(\varepsilon^{1 /(N-2)}|y|\right)\left(W_{r, \Lambda}^{p-1+\varepsilon} \frac{\partial W_{r, \Lambda}}{\partial \Lambda}-\sum_{j=1}^{k} U_{x_{j}, \Lambda}^{p-1} \frac{\partial U_{x_{j}, \Lambda}}{\partial \Lambda}\right) \phi_{r, \Lambda} \\
& \quad+\sum_{j=1}^{k} \int_{B_{*}(0)}\left(K\left(\varepsilon^{1 /(N-2)}|y|\right)-1\right) U_{x_{j}, \Lambda}^{p-1} \frac{\partial U_{x_{j}, \Lambda}}{\partial \Lambda} \phi_{r, \Lambda} \\
= & k \int_{\Omega_{1}} K\left(\varepsilon^{1 /(N-2)}|y|\right)\left(W_{r, \Lambda}^{p-1+\varepsilon} \frac{\partial W_{r, \Lambda}}{\partial \Lambda}-\sum_{j=1}^{k} U_{x_{j}, \Lambda}^{p-1} \frac{\partial U_{x_{j}, \Lambda}}{\partial \Lambda}\right) \phi_{r, \Lambda} \\
& \quad+k \int_{\Omega_{1}}\left(K\left(\varepsilon^{1 /(N-2)}|y|\right)-1\right) U_{x_{1}, \Lambda}^{p-1} \frac{\partial U_{x_{1}, \Lambda}}{\partial \Lambda} \phi_{r, \Lambda},
\end{aligned}
$$

$$
\begin{aligned}
& \left|\int_{\Omega_{1}} K\left(\varepsilon^{1 /(N-2)}|y|\right)\left(W_{r, \Lambda}^{p-1+\varepsilon} \frac{\partial W_{r, \Lambda}}{\partial \Lambda}-\sum_{j=1}^{k} U_{x_{j}, \Lambda}^{p-1} \frac{\partial U_{x_{j}, \Lambda}}{\partial \Lambda}\right) \phi_{r, \Lambda}\right| \\
& \leq C \int_{\Omega_{1}}\left(U_{x_{1}, \Lambda}^{p-1}\left(U_{x_{1}, \Lambda}-P U_{x_{1}, \Lambda}\right)+U_{x_{1}, \Lambda}^{p-1} \sum_{j=2}^{k} U_{x_{j}, \Lambda}+\sum_{j=2}^{k} U_{x_{j}, \Lambda}^{p}\right)\left|\phi_{r, \Lambda}\right| \\
& +O\left(\varepsilon \int_{\Omega_{1}} W_{r, \Lambda}^{p-1} \ln W_{r, \Lambda} \frac{\partial W_{r, \Lambda}}{\partial \Lambda} \phi_{r, \Lambda}\right) \\
& \leq C \varepsilon^{1 /(N-2)(1+\sigma)},
\end{aligned}
$$


and

$$
\begin{aligned}
& \left|\int_{\Omega_{1}}\left(K\left(\varepsilon^{1 /(N-2)}|y|\right)-1\right) U_{x_{1}, \Lambda}^{p-1} \frac{\partial U_{x_{1}, \Lambda}}{\partial \Lambda} \phi_{r, \Lambda}\right| \\
& \leq\left|\int_{|y|-\varepsilon^{-1 /(N-2)} \mid \leq \varepsilon^{-1 /(2(N-2))}}\left(K\left(\varepsilon^{1 /(N-2)}|y|\right)-1\right) U_{x_{1}, \Lambda}^{p-1} \frac{\partial U_{x_{1}, \Lambda}}{\partial \Lambda} \phi_{r, \Lambda}\right| \\
& +\left|\int_{|y|-\varepsilon^{-1 /(N-2)} \mid \geq \varepsilon^{-1 /(2(N-2))}}\left(K\left(\varepsilon^{1 /(N-2)}|y|\right)-1\right) U_{x_{1}, \Lambda}^{p-1} \frac{\partial U_{x_{1}, \Lambda}}{\partial \Lambda} \phi_{r, \Lambda}\right| \\
& \leq C \varepsilon^{1 /(N-2)(1+\sigma)} \text {. }
\end{aligned}
$$

So, we have proved

$$
\begin{aligned}
\int_{B_{*}(0)} & \left(\nabla\left(W_{r, \Lambda}+\phi_{r, \Lambda}\right) \nabla \frac{\partial W_{r, \Lambda}}{\partial \Lambda}-K\left(\varepsilon^{1 /(N-2)}|y|\right)\left(W_{r, \Lambda}+\phi_{r, \Lambda}\right)^{p+\varepsilon} \frac{\partial W_{r, \Lambda}}{\partial \Lambda}\right) \\
= & \int_{B_{*}(0)}\left(\nabla W_{r, \Lambda} \nabla \frac{\partial W_{r, \Lambda}}{\partial \Lambda}-K\left(\varepsilon^{1 /(N-2)}|y|\right) W_{r, \Lambda}^{p+\varepsilon} \frac{\partial W_{r, \Lambda}}{\partial \Lambda}\right)+O\left(k \varepsilon^{(1+\sigma) /(N-2)}\right),
\end{aligned}
$$

and the result follows from Proposition A.1.

Proof of Theorem 1.3. Note (see [Wei and Yan 2013]) that

$$
H\left(\bar{x}_{1}, \bar{x}_{1}\right)=\frac{1}{2^{N-2} d^{N-2}}(1+O(d))
$$

and

$$
\frac{a_{0}}{j^{N}}+O\left(\frac{d}{j^{N-2}}\right) \leq \frac{1}{k^{N-2}} G\left(\bar{x}_{j}, \bar{x}_{1}\right) \leq \frac{a_{1}}{j^{N}}+O\left(\frac{d}{j^{N-2}}\right),
$$

where $a_{1} \geq a_{0}>0$. Hence, we find that there is a constant $B_{4}>0$ such that $\sum_{j=2}^{k} G\left(\bar{x}_{j}, \bar{x}_{1}\right)=k^{N-2}\left(\frac{B_{4}}{\left|\bar{x}_{1}\right|^{N-2}}+O\left(\frac{1}{k^{N-1}}\right)+O(d)\right)=B_{4} k^{N-2}+O\left(k^{N-2} d\right)$.

Consequently, (3-4) and (3-5) are equivalent to

$$
-\frac{A_{1} \varepsilon}{\Lambda^{N-1} d^{N-2}}+\frac{A_{2} k^{N-2} \varepsilon}{\Lambda^{N-1}}+O\left(\varepsilon^{(1+\sigma) /(N-2)}\right)=0
$$

and

$$
-\frac{A_{3} \varepsilon}{\Lambda^{N-2} d^{N-1}}+A_{4}+O\left(\varepsilon^{\sigma /(N-2)}\right)=0
$$

respectively, for positive constants $A_{i}, i=1,2,3,4$. Recall that $d=1-\varepsilon^{1 /(N-2)} r$. Define $\eta=d k$. Thus, (3-6) and (3-7) read

$$
-\frac{A_{1}}{\Lambda^{N-1} \eta^{N-2}}+\frac{A_{2}}{\Lambda^{N-1}}+O\left(\varepsilon^{\sigma /(N-2)}\right)=0
$$


and

$$
-\frac{A_{3}}{\Lambda^{N-2} \eta^{N-1}}+A_{4}+O\left(\varepsilon^{\sigma /(N-2)}\right)=0 .
$$

Let

$$
f_{1}(\eta, \Lambda)=-\frac{A_{1}}{\Lambda^{N-1} \eta^{N-2}}+\frac{A_{2}}{\Lambda^{N-1}}
$$

and

$$
f_{2}(\eta, \Lambda)=-\frac{A_{3}}{\Lambda^{N-2} \eta^{N-1}}+A_{4} .
$$

It is easy to check that $f_{1}=0$ and $f_{2}=0$ have a unique solution

$$
\eta_{0}=\left(\frac{A_{1}}{A_{2}}\right)^{\frac{1}{N-2}}, \quad \Lambda_{0}=\left(\frac{A_{3}}{A_{4} \eta_{0}^{N-1}}\right)^{\frac{1}{N-2}} .
$$

On the other hand, we have

$$
\frac{\partial f_{1}\left(\eta_{0}, \Lambda_{0}\right)}{\partial \Lambda}=0, \quad \frac{\partial f_{2}\left(\eta_{0}, \Lambda_{0}\right)}{\partial \eta}>0,
$$

and

$$
\frac{\partial f_{1}\left(\eta_{0}, \Lambda_{0}\right)}{\partial \eta}>0, \quad \frac{\partial f_{2}\left(\eta_{0}, \Lambda_{0}\right)}{\partial \Lambda}>0 .
$$

Hence the linear operator of $f_{1}=0$ and $f_{2}=0$ at $\left(\eta_{0}, \Lambda_{0}\right)$ is invertible. Therefore, (3-8) and (3-9) have a solution near $\left(\eta_{0}, \Lambda_{0}\right)$.

\section{Appendix A: Energy expansion}

Here and in Appendix B, we assume that

$$
x_{j}=\left(r \cos \frac{2(j-1) \pi}{k}, r \sin \frac{2(j-1) \pi}{k}, 0\right), \quad j=1, \ldots, k,
$$

where $r \in\left[\varepsilon^{-\frac{1}{N-2}}\left(1-r_{0} \varepsilon^{\frac{1}{N-1}}\right), \varepsilon^{-\frac{1}{N-2}}\left(1-r_{1} \varepsilon^{\frac{1}{N-1}}\right)\right]$ and 0 is the zero vector in $\mathbb{R}^{N-2}$.

Let

$$
\bar{x}_{j}=\varepsilon^{\frac{1}{N-2}} x_{j}
$$

Let $G(x, y)$ be the Green function of $-\Delta$ in $B_{1}(0)$ with the Dirichlet boundary and let $H(x, y)$ be the regular part of the Green function.

Recall that

$$
k=\left[\varepsilon^{-\frac{1}{N-1}}\right]
$$

and

$$
W_{r, \Lambda}(y)=\sum_{j=1}^{k} P U_{x_{j}, \Lambda}(y)
$$


where $P U_{x, \Lambda}$ is the solution of (1-5). Moreover,

$$
\phi_{x_{j}, \Lambda}(y)=U_{x_{j}, \Lambda}(y)-P U_{x_{j}, \Lambda}(y)=\frac{\varepsilon H\left(\bar{x}_{j}, \bar{y}\right)}{\Lambda^{\frac{1}{2}(N-2)}}+O\left(\frac{\varepsilon^{N /(N-2)}}{d^{N}}\right),
$$

where $d=1-\left|\bar{x}_{j}\right|=1-\varepsilon^{1 /(N-2)}\left|x_{j}\right|$.

Proposition A.1. We have

$$
\begin{aligned}
\int_{B_{*}(0)}\left(\nabla W_{r, \Lambda} \nabla \frac{\partial W_{r, \Lambda}}{\partial \Lambda}\right. & \left.-K\left(\varepsilon^{1 /(N-2)}|y|\right) W_{r, \Lambda}^{p+\varepsilon} \frac{\partial W_{r, \Lambda}}{\partial \Lambda}\right) \\
& =k B_{1}\left(-\frac{\varepsilon H\left(\bar{x}_{1}, \bar{x}_{1}\right)}{\Lambda^{N-1}}+\sum_{i=2}^{k} \frac{\varepsilon G\left(\bar{x}_{i}, \bar{x}_{1}\right)}{\Lambda^{N-1}}+O\left(\varepsilon^{(1+\sigma) /(N-2)}\right)\right),
\end{aligned}
$$

and

$$
\begin{aligned}
\int_{B_{*}(0)}\left(\nabla W_{r, \Lambda} \nabla \frac{\partial W_{r, \Lambda}}{\partial r}-K\left(\varepsilon^{1 /(N-2)}|y|\right) W_{r, \Lambda}^{p+\varepsilon} \frac{\partial W_{r, \Lambda}}{\partial r}\right) \\
=k\left(\frac{B_{2} \varepsilon}{\Lambda^{N-2}} \frac{\partial H\left(\bar{x}_{1}, \bar{x}_{1}\right)}{\partial r}-B_{3} K^{\prime}(1) \varepsilon^{1 /(N-2)}\right. \\
\left.+\sum_{i=2}^{k} \frac{B_{2} \varepsilon}{\Lambda^{N-2}} \frac{\partial G\left(\bar{x}_{i}, \bar{x}_{1}\right)}{\partial r}+O\left(\varepsilon^{(1+\sigma) /(N-2)}\right)\right),
\end{aligned}
$$

where $B_{1}, B_{2}$ and $B_{3}$ are some positive constants.

Proof. The proof is quite standard now. Here we only prove the first equation. The other one can be obtained similarly.

Using symmetry, we find

$$
\begin{aligned}
I & :=\int_{B_{*}(0)}\left(\nabla W_{r, \Lambda} \nabla \frac{\partial W_{r, \Lambda}}{\partial \Lambda}-K\left(\varepsilon^{1 /(N-2)}|y|\right) W_{r, \Lambda}^{p+\varepsilon} \frac{\partial W_{r, \Lambda}}{\partial \Lambda}\right) \\
& =k\left(p \sum_{i=1}^{k} \int_{B_{*}(0)} P U_{x_{1}, \Lambda}^{p-1} \frac{\partial P U_{x_{1}, \Lambda}}{\partial \Lambda} P U_{x_{i}, \Lambda}-\int_{\Omega_{1}} K\left(\varepsilon^{1 /(N-2)}|y|\right) W_{r, \Lambda}^{p+\varepsilon} \frac{\partial W_{r, \Lambda}}{\partial \Lambda}\right) .
\end{aligned}
$$

It is easy to check that for $y \in \Omega_{1}$,

$$
\begin{aligned}
\frac{\partial}{\partial \Lambda} W_{r, \Lambda}^{p+1}=\frac{\partial}{\partial \Lambda} P U_{x_{1}, \Lambda}^{p+1}+(p+1) \frac{\partial}{\partial \Lambda}\left(P U_{x_{1}, \Lambda}^{p}\right. & \left.\sum_{i=2}^{k} P U_{x_{i}, \Lambda}\right) \\
& +O\left(U_{x_{1}, \Lambda}^{\frac{1}{2}(p+1)}\left(\sum_{i=2}^{k} U_{x_{i}, \Lambda}\right)^{\frac{1}{2}(p+1)}\right) .
\end{aligned}
$$


Thus, we have

$$
\begin{aligned}
& (p+1) \int_{\Omega_{1}} K\left(\varepsilon^{1 /(N-2)}|y|\right) W_{r, \Lambda}^{p+\varepsilon} \frac{\partial W_{r, \Lambda}}{\partial \Lambda} \\
& =\int_{\Omega_{1}} K\left(\varepsilon^{1 /(N-2)}|y|\right) \frac{\partial W_{r, \Lambda}^{p+1}}{\partial \Lambda}+O\left(\varepsilon \int_{\Omega_{1}} W_{r, \Lambda}^{p+1} \ln W_{r, \Lambda}\right) \\
& =\int_{\Omega_{1}} K\left(\varepsilon^{1 /(N-2)}|y|\right) \frac{\partial}{\partial \Lambda} P U_{x_{1}, \Lambda}^{p+1} \\
& \quad+(p+1) \int_{\Omega_{1}} K\left(\varepsilon^{1 /(N-2)}|y|\right) \frac{\partial}{\partial \Lambda}\left(P U_{x_{1}, \Lambda}^{p} \sum_{i=2}^{k} P U_{x_{i}, \Lambda}\right) \\
& \quad+O\left(\int_{\Omega_{1}} U_{x_{1}, \Lambda}^{\frac{1}{2}(p+1)}\left(\sum_{i=2}^{k} U_{x_{i}, \Lambda}\right)^{\frac{1}{2}(p+1)}\right)+O\left(\varepsilon \int_{\Omega_{1}} W_{r, \Lambda}^{p+1} \ln W_{r, \Lambda}\right) .
\end{aligned}
$$

Note that for $y \in \Omega_{1},\left|y-x_{i}\right| \geq\left|y-x_{1}\right|$. Using (2-11), we see that for $t \in(1, N-2)$,

$$
\sum_{i=2}^{k} U_{x_{i}, \Lambda} \leq \frac{C}{\left(1+\left|y-x_{1}\right|\right)^{N-2-t}} \sum_{i=2}^{k} \frac{1}{\left|x_{i}-x_{1}\right|^{t}} .
$$

If we take $t$ close to $N-2$, then

$$
\int_{\Omega_{1}} U_{x_{1}, \Lambda}^{\frac{1}{2}(p+1)}\left(\sum_{i=2}^{k} U_{x_{i}, \Lambda}\right)^{\frac{1}{2}(p+1)}=O\left(\left(k \varepsilon^{1 /(N-2)}\right)^{N t /(N-2)}\right)=O\left(\varepsilon^{(1+\sigma) /(N-2)}\right) .
$$

Moreover, it is easy to show that

$$
\varepsilon \int_{\Omega_{1}} W_{r, \Lambda}^{p+1} \ln W_{r, \Lambda}=O(\varepsilon)
$$

As a result, we obtain

$$
\begin{aligned}
& I=k\left(-\int_{\Omega_{1}} K\left(\varepsilon^{1 /(N-2)}|y|\right) P U_{x_{1}, \Lambda}^{p} \frac{\partial P U_{x_{1}, \Lambda}}{\partial \Lambda}\right. \\
& \quad-\sum_{i=2}^{k} \int_{\Omega_{1}} K\left(\varepsilon^{1 /(N-2)}|y|\right) P U_{x_{1}, \Lambda}^{p} \frac{\partial P U_{x_{i}, \Lambda}}{\partial \Lambda} \\
& \quad+p \sum_{i=2}^{k} \int_{\Omega_{1}}\left(1-K\left(\varepsilon^{1 /(N-2)}|y|\right)\right) P U_{x_{1}, \Lambda}^{p-1} \frac{\partial P U_{x_{1}, \Lambda}}{\partial \Lambda} P U_{x_{i}, \Lambda} \\
& \left.\quad+p \sum_{i=2}^{k} \int_{B_{*}(0) \backslash \Omega_{1}} P U_{x_{1}, \Lambda}^{p-1} \frac{\partial P U_{x_{1}, \Lambda}}{\partial \Lambda} P U_{x_{i}, \Lambda}+O\left(\varepsilon^{(1+\sigma) /(N-2)}\right)\right) .
\end{aligned}
$$


On the other hand,

$$
\begin{aligned}
\int_{\Omega_{1}} K\left(\varepsilon^{1 /(N-2)}|y|\right) P U_{x_{1}, \Lambda}^{p} \frac{\partial P U_{x_{1}, \Lambda}}{\partial \Lambda} \\
=\int_{\Omega_{1}} P U_{x_{1}, \Lambda}^{p} \frac{\partial P U_{x_{1}, \Lambda}}{\partial \Lambda}+\int_{\Omega_{1}}\left(K\left(\varepsilon^{1 /(N-2)}|y|\right)-1\right) P U_{x_{1}, \Lambda}^{p} \frac{\partial P U_{x_{1}, \Lambda}}{\partial \Lambda} \\
=\int_{\Omega_{1}} P U_{x_{1}, \Lambda}^{p} \frac{\partial P U_{x_{1}, \Lambda}}{\partial \Lambda} \\
\quad+\int_{\Omega_{1}}\left(K\left(\left|\bar{x}_{1}\right|\right)-1\right) P U_{x_{1}, \Lambda}^{p} \frac{\partial P U_{x_{1}, \Lambda}}{\partial \Lambda}+O\left(\varepsilon^{(1+\sigma) /(N-2)}\right) \\
=\int_{\Omega_{1}} P U_{x_{1}, \Lambda}^{p} \frac{\partial P U_{x_{1}, \Lambda}}{\partial \Lambda}-K^{\prime}(1) d \int_{\Omega_{1}} P U_{x_{1}, \Lambda}^{p} \frac{\partial P U_{x_{1}, \Lambda}}{\partial \Lambda}+O\left(\varepsilon^{(1+\sigma) /(N-2)}\right) \\
=-\int_{\Omega_{1}} U_{x_{1}, \Lambda}^{p} \frac{\partial \phi_{x_{1}, \Lambda}}{\partial \Lambda}-p \int_{\Omega_{1}} U_{x_{1}, \Lambda}^{p} \frac{\partial U_{x_{1}, \Lambda}}{\partial \Lambda} \phi_{x_{1}, \Lambda}+O\left(\varepsilon^{(1+\sigma) /(N-2)}\right) \\
=\frac{B_{1} \varepsilon H\left(\bar{x}_{1}, \bar{x}_{1}\right)}{\Lambda^{N-1}}+O\left(\varepsilon^{(1+\sigma) /(N-2)}\right)
\end{aligned}
$$

and

$$
\begin{aligned}
\int_{\Omega_{1}} K\left(\varepsilon^{1 /(N-2)}|y|\right) P U_{x_{1}, \Lambda}^{p} \frac{\partial P U_{x_{i}, \Lambda}}{\partial \Lambda} \\
=\int_{\Omega_{1}} P U_{x_{1}, \Lambda}^{p} \frac{\partial P U_{x_{i}, \Lambda}}{\partial \Lambda}+\int_{\Omega_{1}}\left(K\left(\varepsilon^{1 /(N-2)}|y|\right)-1\right) P U_{x_{1}, \Lambda}^{p} \frac{\partial P U_{x_{i}, \Lambda}}{\partial \Lambda} \\
=\int_{\Omega_{1}} U_{x_{1}, \Lambda}^{p} \frac{\partial U_{x_{i}, \Lambda}}{\partial \Lambda}-\int_{\Omega_{1}} U_{x_{1}, \Lambda}^{p} \frac{\partial \phi_{x_{i}, \Lambda}}{\partial \Lambda}+O\left(\varepsilon^{(1+\sigma) /(N-2)}\right) \\
=-\frac{B_{1} \varepsilon G\left(\bar{x}_{i}, \bar{x}_{1}\right)}{\Lambda^{N-1}}+O\left(\varepsilon^{(1+\sigma) /(N-2)}\right) .
\end{aligned}
$$

Other terms can be estimated similarly. Thus, the result follows.

\section{Appendix B: Basic estimates}

In this section, we will give some basic estimates used in the reduction procedure. We will use the same constant $C>0$ to denote the different constants.

Lemma B.1. Let $g_{i j}=1 /\left(\left(1+\left|y-x_{i}\right|\right)^{\alpha}\left(1+\left|y-x_{j}\right|\right)^{\beta}\right)$ for each fixed $i$ and $j$, $i \neq j$, where $\alpha \geq 1$ and $\beta \geq 1$ are two constants. Then for any $0<\sigma \leq \min (\alpha, \beta)$, there is a constant $C>0$ such that

$$
g_{i j}(y) \leq \frac{C}{\left|x_{i}-x_{j}\right|^{\sigma}}\left(\frac{1}{\left(1+\left|y-x_{i}\right|\right)^{\alpha+\beta-\sigma}}+\frac{1}{\left(1+\left|y-x_{j}\right|\right)^{\alpha+\beta-\sigma}}\right) .
$$


Lemma B.2. For any constant $0<\sigma<N-2$, there is a constant $C>0$ such that

$$
\int_{\mathbb{R}^{N}} \frac{1}{|y-z|^{N-2}} \frac{1}{(1+|z|)^{2+\sigma}} d z \leq \frac{C}{(1+|y|)^{\sigma}} .
$$

The proofs of the above two lemmas can be found in [Wei and Yan 2010b].

Lemma B.3. Suppose that $\varepsilon>0$ and $N \geq 4$. Then there is a small $\vartheta>0$ such that

$$
\begin{aligned}
& \int_{\mathbb{R}^{N}} \frac{1}{|y-z|^{N-2}} W_{r, \Lambda}^{p-1+\varepsilon}(z) \sum_{j=1}^{k} \frac{1}{\left(1+\left|z-x_{j}\right|\right)^{\frac{1}{2}(N-2)+\tau}} d z \\
& \quad \leq C \sum_{j=1}^{k} \frac{1}{\left(1+\left|z-x_{j}\right|\right)^{\frac{1}{2}(N-2)+\tau+\vartheta}} .
\end{aligned}
$$

Proof. This is similar to the proof of Lemma B.3 in [Wei and Yan 2010b]. So we just sketch it. Note that

$$
W_{r, \Lambda}(z) \leq C \sum_{j=1}^{k} \frac{1}{\left(1+\left|z-x_{j}\right|\right)^{N-2}} .
$$

As in [Wei and Yan 2010b], for $y \in \Omega_{1}$ we have $W_{r, \Lambda}(z) \leq \frac{C}{\left(1+\left|z-x_{1}\right|\right)^{N-2-\tau_{1}}}$, where $0<\tau_{1} \leq \frac{1}{2}(N-2)$. Thus,

$$
W_{r, \Lambda}^{p-1+\varepsilon}(z) \leq \frac{C}{\left(1+\left|z-x_{1}\right|\right)^{4-\frac{4 \tau_{1}}{N-2}+\left(N-2-\tau_{1}\right) \varepsilon}} .
$$

By virtue of Lemma B.1, for $y \in \Omega_{1}$ we get

$$
\begin{aligned}
& W_{r, \Lambda}^{p-1+\varepsilon}(z) \sum_{j=1}^{k} \frac{1}{\left(1+\left|z-x_{j}\right|\right)^{\frac{1}{2}(N-2)+\tau}} \\
& \leq \frac{C}{\left(1+\left|z-x_{1}\right|\right)^{\frac{1}{2}(N+6)+\tau-\frac{4 \tau_{1}}{N-2}+\left(N-2-\tau_{1}\right) \varepsilon}} \\
& +\sum_{j=2}^{k} \frac{C}{\left(1+\left|z-x_{1}\right|\right)^{4-\frac{4 \tau_{1}}{N-2}+\left(N-2-\tau_{1}\right) \varepsilon}} \frac{1}{\left(1+\left|z-x_{j}\right|\right)^{\frac{1}{2}(N-2)+\tau}} \\
& \leq \frac{C}{\left(1+\left|z-x_{1}\right|\right)^{\frac{1}{2}(N+6)+\tau-\frac{4 \tau_{1}}{N-2}+\left(N-2-\tau_{1}\right) \varepsilon}} \\
& +\frac{C}{\left(1+\left|z-x_{1}\right|\right)^{\frac{1}{2}(N+6)+\tau-\frac{N+2}{N-2} \tau_{1}+\left(N-2-\tau_{1}\right) \varepsilon}} \sum_{j=2}^{k} \frac{1}{\left|x_{j}-x_{1}\right|^{\tau_{1}}} \\
& \leq \frac{C}{\left(1+\left|z-x_{1}\right|\right)^{\frac{1}{2}(N+6)+\tau-\frac{N+2}{N-2} \tau_{1}+\left(N-2-\tau_{1}\right) \varepsilon} .}
\end{aligned}
$$


Thus, we can obtain

$$
\begin{gathered}
\int_{\Omega_{1}} \frac{1}{|y-z|^{N-2}} W_{r, \Lambda}^{p-1+\varepsilon}(z) \sum_{j=1}^{k} \frac{1}{\left(1+\left|z-x_{j}\right|\right)^{\frac{1}{2}(N-2)+\tau}} d z \\
\quad \leq \int_{\Omega_{1}} \frac{1}{|y-z|^{N-2}} \frac{C}{\left(1+\left|z-x_{1}\right|\right)^{\frac{1}{2}(N+6)+\tau-\frac{N+2}{N-2} \tau_{1}+\left(N-2-\tau_{1}\right) \varepsilon}} d z \\
\quad \leq \frac{C}{\left(1+\left|z-x_{1}\right|\right)^{\frac{1}{2}(N+2)+\tau-\frac{N+2}{N-2} \tau_{1}+\left(N-2-\tau_{1}\right) \varepsilon}} .
\end{gathered}
$$

As a result, for $\tau_{1}$ satisfying $2-(N+2) /(N-2) \tau_{1}>0$, we find that

$$
\begin{aligned}
\int_{\mathbb{R}^{N}} \frac{1}{|y-z|^{N-2}} W_{r, \Lambda}^{p-1+\varepsilon}(z) \sum_{j=1}^{k} \frac{1}{\left(1+\left|z-x_{j}\right|\right)^{\frac{1}{2}(N-2)+\tau}} d z \\
\quad=\sum_{i=1}^{k} \int_{\Omega_{i}} \frac{1}{|y-z|^{N-2}} W_{r, \Lambda}^{p-1+\varepsilon}(z) \sum_{j=1}^{k} \frac{1}{\left(1+\left|z-x_{j}\right|\right)^{\frac{1}{2}(N-2)+\tau}} d z \\
\quad \leq \sum_{i=1}^{k} \frac{C}{\left(1+\left|z-x_{1}\right|\right)^{\frac{1}{2}(N+2)+\tau-\frac{N+2}{N-2} \tau_{1}+\left(N-2-\tau_{1}\right) \varepsilon}} \\
\leq C \sum_{j=1}^{k} \frac{1}{\left(1+\left|z-x_{j}\right|\right)^{\frac{1}{2}(N-2)+\tau+\vartheta}}
\end{aligned}
$$

\section{Acknowledgements}

The authors would like to thank the referee very much for the careful reading of the paper and valuable comments, including pointing out Remark 1.5. Z. Liu was supported by funds from the NSFC (No. 11426088, No. 11501166); S. Peng was partially supported by funds from the NSFC (No. 11125101, No. 11571130).

\section{References}

[Ben Ayed et al. 2003] M. Ben Ayed, K. El Mehdi, O. Rey, and M. Grossi, "A nonexistence result of single peaked solutions to a supercritical nonlinear problem”, Commun. Contemp. Math. 5:2 (2003), 179-195. MR 2004k:35140 Zbl 1066.35035

[Byeon and Wang 2005] J. Byeon and Z.-Q. Wang, "On the Hénon equation: asymptotic profile of ground states, II”, J. Differential Equations 216:1 (2005), 78-108. MR 2006j:35068 Zbl 1114.35070

[Byeon and Wang 2006] J. Byeon and Z.-Q. Wang, "On the Hénon equation: asymptotic profile of ground states, I”, Ann. Inst. H. Poincaré Anal. Non Linéaire 23:6 (2006), 803-828. MR 2007g:35046 Zbl 1114.35071

[Cao and Peng 2003] D. Cao and S. Peng, "The asymptotic behaviour of the ground state solutions for Hénon equation”, J. Math. Anal. Appl. 278:1 (2003), 1-17. MR 2003m:35062 Zbl 1086.35036 
[Cao et al. 2009] D. Cao, S. Peng, and S. Yan, "Asymptotic behaviour of ground state solutions for the Hénon equation”, IMA J. Appl. Math. 74:3 (2009), 468-480. MR 2010d:35088 Zbl 1169.35377

[Gidas et al. 1979] B. Gidas, W. M. Ni, and L. Nirenberg, "Symmetry and related properties via the maximum principle”, Comm. Math. Phys. 68:3 (1979), 209-243. MR 80h:35043 Zbl 0425.35020

[Gladiali and Grossi 2012] F. Gladiali and M. Grossi, "Supercritical elliptic problem with nonautonomous nonlinearities", J. Differential Equations 253:9 (2012), 2616-2645. MR 2959382 Zbl 1266.35105

[Gladiali et al. 2013] F. Gladiali, M. Grossi, and S. L. N. Neves, "Nonradial solutions for the Hénon equation in $\mathbb{R}^{N}$ ", Adv. Math. 249 (2013), 1-36. MR 3116566 Zbl 06296090

[Hénon 1973] M. Hénon, "Numerical experiments on the stability of spherical stellar systems", Astron. Astrophys. 24 (1973), 229-238.

[Hirano 2009] N. Hirano, "Existence of positive solutions for the Hénon equation involving critical Sobolev terms", J. Differential Equations 247:5 (2009), 1311-1333. MR 2010f:35121 Zbl 1176. 35083

[Li and Peng 2009] S. Li and S. Peng, "Asymptotic behavior on the Hénon equation with supercritical exponent”, Sci. China Ser. A 52:10 (2009), 2185-2194. MR 2011b:35159 Zbl 1184.35136

[Ni 1982] W. M. Ni, "A nonlinear Dirichlet problem on the unit ball and its applications", Indiana Univ. Math. J. 31:6 (1982), 801-807. MR 84b:35051 Zbl 0515.35033

[Peng 2006] S. Peng, "Multiple boundary concentrating solutions to Dirichlet problem of Hénon equation”, Acta Math. Appl. Sin. Engl. Ser. 22:1 (2006), 137-162. MR 2006k:35099 Zbl 1153.35325

[Peng and Wang 2013] S. Peng and Z.-Q. Wang, "Segregated and synchronized vector solutions for nonlinear Schrödinger systems", Arch. Ration. Mech. Anal. 208:1 (2013), 305-339. MR 3021550 Zbl 1260.35211

[del Pino et al. 2003] M. del Pino, P. Felmer, and M. Musso, "Two-bubble solutions in the supercritical Bahri-Coron's problem”, Calc. Var. Partial Differential Equations 16:2 (2003), 113-145. MR 2004a:35079 Zbl 1142.35421

[Pistoia and Serra 2007] A. Pistoia and E. Serra, "Multi-peak solutions for the Hénon equation with slightly subcritical growth”, Math. Z. 256:1 (2007), 75-97. MR 2008b:35091 Zbl 1134.35047

[Pohožaev 1965] S. I. Pohožaev, "On the eigenfunctions of the equation $\Delta u+\lambda f(u)=0$ ", Dokl. Akad. Nauk SSSR 165 (1965), 36-39. In Russian; translated in Sov. Math. Dokl. 6 (1965), 1408-1411. MR 33 \#411 Zbl 0141.30202

[Serra 2005] E. Serra, "Non radial positive solutions for the Hénon equation with critical growth", Calc. Var. Partial Differential Equations 23:3 (2005), 301-326. MR 2006f:35100 Zbl 1207.35147

[Smets et al. 2002] D. Smets, M. Willem, and J. Su, "Non-radial ground states for the Hénon equation”, Commun. Contemp. Math. 4:3 (2002), 467-480. MR 2003g:35086 Zbl 1160.35415

[Wei and Yan 2010a] J. Wei and S. Yan, "Infinitely many positive solutions for the nonlinear Schrödinger equations in $\mathbb{R}^{N}$,, Calc. Var. Partial Differential Equations 37:3-4 (2010), 423-439. MR 2011c:35065 Zbl 1189.35106

[Wei and Yan 2010b] J. Wei and S. Yan, "Infinitely many solutions for the prescribed scalar curvature problem on $\mathbb{S}^{N}$ ”, J. Funct. Anal. 258:9 (2010), 3048-3081. MR 2011g:35119 Zbl 1209.53028

[Wei and Yan 2011] J. Wei and S. Yan, "Infinitely many positive solutions for an elliptic problem with critical or supercritical growth", J. Math. Pures Appl. (9) 96:4 (2011), 307-333. MR 2832637 Zbl 1253.31008

[Wei and Yan 2013] J. Wei and S. Yan, "Infinitely many nonradial solutions for the Hénon equation with critical growth”, Rev. Mat. Iberoam. 29:3 (2013), 997-1020. MR 3090144 Zbl 1277.35330 
Received November 6, 2014. Revised May 10, 2015.

ZHONGYUAN LIU

SCHOOL OF MATHEMATICS AND STATISTICS

HENAN UNIVERSITY

KAIFENG, HENAN 475004

CHINA

liuzy@henu.edu.cn

ShuANGJIE PENG

SCHOOL OF MATHEMATICS AND STATISTICS

Hubei Key Laboratory of Mathematical Physics

CENTRAL CHINA Normal UNIVERSiTy

WuHAN, HuBEI 430079

CHINA

sjpeng@mail.ccnu.edu.cn 


\title{
PACIFIC JOURNAL OF MATHEMATICS
}

\author{
msp.org/pjm
}

Founded in 1951 by E. F. Beckenbach (1906-1982) and F. Wolf (1904-1989)

\section{EDITORS}

Don Blasius (Managing Editor)

Department of Mathematics

University of California

Los Angeles, CA 90095-1555

blasius@math.ucla.edu

\author{
Paul Balmer \\ Department of Mathematics \\ University of California \\ Los Angeles, CA 90095-1555 \\ balmer@math.ucla.edu \\ Robert Finn \\ Department of Mathematics \\ Stanford University \\ Stanford, CA 94305-2125 \\ finn@math.stanford.edu \\ Sorin Popa \\ Department of Mathematics \\ University of California \\ Los Angeles, CA 90095-1555 \\ popa@math.ucla.edu
}

\author{
Vyjayanthi Chari \\ Department of Mathematics \\ University of California \\ Riverside, CA 92521-0135 \\ chari@math.ucr.edu \\ Kefeng Liu \\ Department of Mathematics \\ University of California \\ Los Angeles, CA 90095-1555 \\ liu@math.ucla.edu \\ Jie Qing \\ Department of Mathematics \\ University of California \\ Santa Cruz, CA 95064 \\ qing@ cats.ucsc.edu
}

\section{PRODUCTION}

Silvio Levy, Scientific Editor, production@msp.org

\section{SUPPORTING INSTITUTIONS}

ACADEMIA SINICA, TAIPEI

CALIFORNIA INST. OF TECHNOLOGY

INST. DE MATEMÁTICA PURA E APLICADA

KEIO UNIVERSITY

MATH. SCIENCES RESEARCH INSTITUTE

NEW MEXICO STATE UNIV.

OREGON STATE UNIV.

\author{
STANFORD UNIVERSITY \\ UNIV. OF BRITISH COLUMBIA \\ UNIV. OF CALIFORNIA, BERKELEY \\ UNIV. OF CALIFORNIA, DAVIS \\ UNIV. OF CALIFORNIA, LOS ANGELES \\ UNIV. OF CALIFORNIA, RIVERSIDE \\ UNIV. OF CALIFORNIA, SAN DIEGO \\ UNIV. OF CALIF., SANTA BARBARA
}

\author{
Daryl Cooper \\ Department of Mathematics \\ University of California \\ Santa Barbara, CA 93106-3080 \\ cooper@math.ucsb.edu \\ Jiang-Hua Lu \\ Department of Mathematics \\ The University of Hong Kong \\ Pokfulam Rd., Hong Kong \\ jhlu@maths.hku.hk \\ Paul Yang \\ Department of Mathematics \\ Princeton University \\ Princeton NJ 08544-1000 \\ yang@math.princeton.edu
}

These supporting institutions contribute to the cost of publication of this Journal, but they are not owners or publishers and have no responsibility for its contents or policies.

See inside back cover or msp.org/pjm for submission instructions.

The subscription price for 2016 is US $\$ 440 /$ year for the electronic version, and $\$ 600 /$ year for print and electronic.

Subscriptions, requests for back issues and changes of subscribers address should be sent to Pacific Journal of Mathematics, P.O. Box 4163, Berkeley, CA 94704-0163, U.S.A. The Pacific Journal of Mathematics is indexed by Mathematical Reviews, Zentralblatt MATH, PASCAL CNRS Index, Referativnyi Zhurnal, Current Mathematical Publications and Web of Knowledge (Science Citation Index).

The Pacific Journal of Mathematics (ISSN 0030-8730) at the University of California, c/o Department of Mathematics, 798 Evans Hall \#3840, Berkeley, CA 94720-3840, is published twelve times a year. Periodical rate postage paid at Berkeley, CA 94704, and additional mailing offices. POSTMASTER: send address changes to Pacific Journal of Mathematics, P.O. Box 4163, Berkeley, CA 94704-0163.

PJM peer review and production are managed by EditFLOW ${ }^{\circledR}$ from Mathematical Sciences Publishers.

\section{PUBLISHED BY}

\section{mathematical sciences publishers \\ nonprofit scientific publishing}

http://msp.org/

(C) 2016 Mathematical Sciences Publishers 


\section{PACIFIC JOURNAL OF MATHEMATICS}

Volume $280 \quad$ No. $1 \quad$ January 2016

Stable capillary hypersurfaces in a wedge

JAIGYOUNG CHOE and MIYUKI KoISO

The Chern-Simons invariants for the double of a compression body

DAVID L. DUNCAN

Compactness and the Palais-Smale property for critical Kirchhoff equations in

closed manifolds

EMMANUEL HeBEy

On the equivalence of the definitions of volume of representations

SUNGWOON KIM

Strongly positive representations of even GSpin groups

YEANSU KIM

An Orlik-Raymond type classification of simply connected 6-dimensional torus manifolds with vanishing odd-degree cohomology

\section{SHINTARÔ KUROKI}

Solutions with large number of peaks for the supercritical Hénon equation

Zhongyuan LiU and ShuangJie Peng

Effective divisors on the projective line having small diagonals and small heights and 141 their application to adelic dynamics

YÛSUKE OKUYAMA

Computing higher Frobenius-Schur indicators in fusion categories constructed from inclusions of finite groups

\section{Peter Schauenburg}

Chordal generators and the hydrodynamic normalization for the unit ball

SEBASTIAN SCHLEISSINGER

On a question of A. Balog

ILYA D. SHKREDOV

Uniqueness result on nonnegative solutions of a large class of differential inequalities 241 on Riemannian manifolds

YUHUA SUN

Correction to "Closed orbits of a charge in a weakly exact magnetic field"

WILL J. MERRY 